\title{
How Has Internet Addiction Research Evolved Since the Advent of Internet Gaming Disorder? An Overview of Cyberaddictions from a Psychological Perspective
}

\author{
Olatz Lopez-Fernandez ${ }^{1}$
}

Published online: 26 July 2015

(C) Springer International Publishing AG 2015

\begin{abstract}
During the past two decades, Internet addiction (IA) has been the most commonly used term in research into online activities and their influence on the development of behavioral addictions. The aim of this review is to assess the impact of the concept of Internet gaming disorder (IGD), proposed by the American Psychiatric Association, on the scientific literature regarding IA. It presents a bibliometric analysis of the IA literature starting from the time IGD was first proposed, with the objective of observing and comparing the topics that have arisen during this period among the different IA themes researched. The findings demonstrate a steady evolution, particularly regarding publications related to the general aspects of IA: its clinical component, its prevalence and psychometric measures, the growing interest in the contextual factors promoting this addictive behavior, scientific progress in its conceptualization based on existing theoretical models, and neuropsychological studies. Nevertheless, many of the studies $(22 \%)$ focus on specific IA behaviors and show heterogeneity among the cyberaddictions, with online gaming (related to IGD) most common, followed by cybersex and social networking. Although research on the general concept of IA continues, investigators have begun to pay attention to the diverse spectrum of specific cyberaddictions and their psychological components.
\end{abstract}

This article is part of the Topical Collection on Technology and Addiction

Olatz Lopez-Fernandez

olatz.lopez@uclouvain.be; lopez.olatz@gmail.com

1 Laboratory for Experimental Psychopathology, Psychological Sciences Research Institute, Université Catholique de Louvain, 10 Place du Cardinal Mercier, 1348 Louvain-La-Neuve, Belgium
Keywords Behavioral addiction · Internet addiction · Cyberaddictions · Internet gaming disorder

\section{Introduction}

During the past two decades, Internet addiction (IA) has been the most commonly used term among researchers examining online activities and their influence on the development of general or specific behavioral addictions. IA usually is defined as an individual's inability to control his or her Internet use, which might lead to the development (and maintenance) of addictive symptomatology, functional impairment, and, in some users, comorbidity. The term is thought to have been coined around the mid-1990s [1•]; expert researchers, such as Griffiths [2•], argued for the existence of technologic (behavioral) addictions in 1995. Since then, scientific research on the topic has grown substantially [3]; by 2005, IA had become the most intensely studied technologic addiction, followed by addiction to video games (including online games), online gambling, and cell phones. However, its increase is a subject of academic, scientific, and clinical debate.

A crucial step forward was taken in May 2013 when the American Psychiatric Association (APA) proposed including the term Internet gaming disorder (IGD) in the appendix of the fifth edition of the Diagnostic and Statistical Manual of Mental Disorders (DSM-5) as a condition warranting more empirical and clinical research [4•]. The recognition of this new phenomenon as a possible mental disorder has had a strong impact on the international scientific community. Petry and colleagues $[5 \cdot \bullet$, $6 \cdot 0$ ] established guidelines based on nine potential criteria for IGD, as well as their underlying significance; offered a common method to assess the condition via these nine 
items, with a conservative cutoff point of five; and translated the guidelines into ten languages to facilitate crosscultural studies. Researchers in the field of behavioral addictions immediately expressed their opinion [7-12], with their main concerns about IGD focused on the proposed criteria (e.g., on symptomatology such as preoccupation or withdrawal, the frequency or intensity of gaming, or the wording of items). Thus, a new debate in the field of IA arose. On one hand, the dissemination of IGD criteria in several languages, which has been valuable in promoting the global study of this phenomenon [7], and the attempts to generate more consistency in IGD research generally are considered achievements [8]. On the other hand, however, some experts argue that the new DSM-5 criteria add confusion rather than consensus [9-12] and do not help in overcoming the difficulties related to the definition and recognition of this disorder. The lack of a clear conceptualization has resulted in a multiplicity of strategies to assess IA, with inconsistent criteria and a lack of comparability [13].

Griffiths and Szabo [14•] highlighted one of the longstanding debates in the IA field - whether the addiction is to the Internet in general (i.e., as a medium) or to a specific online activity conducted through it (i.e., content and applications) - suggesting that IA appears to be a specific behavior in the online environment. Similarly, Petry et al. [15•], in observing the conceptual confusion hindering the study of IGD [7-10], stated that the medium through which one accesses games is not important for diagnosis and included offline games with those associated with online or Internet access (clearly distinguishing it from gambling disorder). Therefore, the term technologic addictions [2•] (commonly used in the English literature) or cyberaddictive spectrum [16] (used chiefly in the French literature) may be understood as covering the original term Internet addiction, addressing both addiction to specific technologies (e.g., smartphones) and to addictive behavior carried out through genuinely online activities and applications (e.g., social networking and Facebook). Laconi et al. [17] recently observed the heterogeneity of general and specific online addictions in relation to psychological symptoms, finding higher levels of depression in cybersex addicts and less self-esteem and satisfaction with life among online gamblers and general Internet addicts.

Therefore, this review examines the impact of the introduction of the concept of IGD on scientific research in IA from a psychological perspective, in order to observe indirectly how this controversial field of research is advancing. A bibliometric analysis was performed on the IA literature published since the concept of IGD was introduced in May 2013 [4•], with a twofold aim: (i) to compare the psychological topics that have arisen during this period concerning particular cyberaddictions and (ii) to describe the main topics addressed in IA research.

\section{Literature Review}

\section{Search Strategy}

A literature review was performed by using the advanced search option in the PsycINFO database, the leading academic search engine in psychological science. The first phase of this search included a search equation with terms commonly used in the field of IA (("internet addict*") OR ("internet problem*" OR "internet pathologic*") OR ("internet excessiv*" OR "internet abuse")). Thus, the search was limited to peer-reviewed literature (editorial, journal article, and peer-reviewed journal "record-type" categories) published from May 2013 [4•] to December 2014. A total of 314 documents were obtained; duplicates were avoided by using the ProQuest service (Fig. 1).

These publications appeared in journals related to addictions, specifically behavioral addictions, including Computers in Human Behavior ( $n=37)$; Cyberpsychology, Behavior, and Social Networking $(n=26)$; Journal of Behavioral Addictions $(n=16) ;$ International Journal of Child and Adolescent Health ( $n=16)$; and Addictive Behaviors ( $n=14)$. The results selected were classified by the database as "Behavior disorders and antisocial behavior" $(n=161)$, "Substance abuse and addiction" $(n=45)$, "Psychological and physical disorders" $(n=25)$, "Clinical psychological testing" $(n=24)$, "Psychological disorders" $(n=13)$, and other categories. Based on the participants of these studies, most were performed in adults ( $n=196)$, adolescents (13 to 17 year olds; $n=128$ ), or young adults (18 to 29 year olds; $n=118$ ) and in healthy populations (i.e., very few in outpatients $(n=10)$ or inpatients $(n=4))$. From a methodologic perspective, empirical studies were the most common $(n=236)$, followed by quantitative studies $(n=232)$. Other research methods in the "Methodology" category, such as literature reviews $(n=26)$, brain imaging studies $(n=15)$, and longitudinal $(n=7)$ or qualitative $(n=7)$ studies, were relatively rare.

\section{May 2013 - December 2014 (months)}

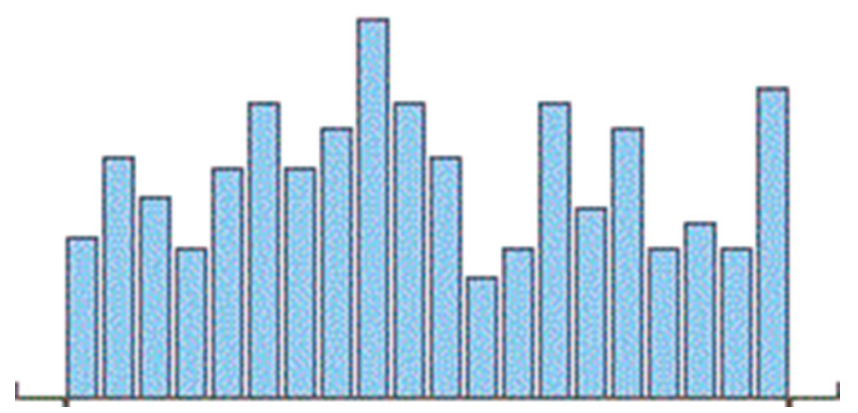

Fig. 1 PsycINFO search. The bars represent the volume of documents published each month from May 2013 to December 2014 
Almost all the studies were published in English $(n=281)$, but nine in Chinese, eight in German, four in Spanish, and three in French. The pattern of publication during this period was quite regular $(M=15.7$; rank $=19$; from 8 in April 2014 to 27 in January 2014), although the number of publications during 2014 was low. Among these publications, 65 included the term Internet gaming disorder.

In the third phase, the search was refined manually. All the titles and abstracts were read to select the most appropriate articles: (i) those clearly related to cyberaddictions (some papers on substance addiction research done via internet surveys were omitted) and (ii) those meeting strictly selected criteria (e.g., book reviews, comments, and replies were removed). In total, 257 papers were retained $(90.8 \%$ of peer-reviewed publications refined), 15 (5.8\% of peer-reviewed papers retained) of which included the term Internet gaming disorder in the main information (i.e., title, abstract, or keywords) and were removed.

\section{Strategy for Analyzing the Content of Internet Addiction Papers}

A thematic content analysis was performed, paying attention to the topics that emerged from the 242 papers not related directly to IGD. In order of frequency, there were seven categories:

1. Specific cyberaddiction studies $(n=69)$, focusing on a particular excessive online activity, such as:

(a) online (video) gaming $(n=26)$

(b) cybersex $(n=16)$

(c) social networking $(n=16)$

(d) online gambling $(n=7)$

(e) online shopping $(n=4)$

2. Clinical studies $(n=61)$, performed in clinical settings with patients or outpatients or those studying IA's relationship to specific psychopathologies, symptomatology, or underlying psychological mechanisms

3. Prevalence studies $(n=39)$, which, e.g., estimate the proportion of potential addicts among high school or college students from different countries or analyze protective or risk factors

4. Contextual factor studies $(n=24)$, focusing on external factors influencing IA, e.g., family, social environment (real or virtual), or religion

5. IA conceptualization studies $(n=22)$, focusing on the general concept of IA, proposing models, or examining relationships to other problems

6. Psychometric studies $(n=16)$, adapting and validating scales for different languages and countries
7. Neuropsychological studies $(n=11)$, applying different techniques of neuroimaging or other neurophysiologic explorations to studying IA

Therefore, $71.5 \%(n=173)$ of the papers retained were related to generalized IA.

The inductive system used to extract the categories was exhaustive and mutually exclusive. Nevertheless, some of the papers were not categorized initially; for example, the "Psychometric development of the Problematic Pornography Use Scale" [18] could be classified in two categories "cybersex" or "psychometric studies." In this case, the cybersex category was chosen because of the interest in highlighting the cyberaddictive spectrum researched since the definition of IGD to observe its heterogeneity.

\section{Preliminary Results from Research Areas of Internet Addiction Production}

\section{Main Findings for Specific Cyberaddictions}

Online (video) game addiction was the most prevalent cyberaddiction, with studies investigating motivational (e.g., the achievement of success, the creation of social relationships, the immersion in an ideal environment [19]), clinical (e.g., mood and anxiety disorders, depressive symptoms and suicidal thoughts, cognitive distortions, academic failure and functional impairment [19-21]), and personality factors (e.g., avoidance, borderline, compulsive-impulsive disorders [19, 20]). The video games most studied for their addictive components were massively multiplayer online role-playing games (MMORPGs) [19, 20, 22], although other genres have been studied recently (i.e., first-person shooter (FPS) and strategy games) [23-25]. Several cognitive processes (e.g., rumination, short-term thinking, risky decisionmaking, escapism, social outlet seeking, and search for reward) have been identified as predictors of online gaming addiction [21, 24, 26]. With regard to treatment, the most effective approach reported was cognitive-behavioral therapy (CBT) [19, 21, 27]. Lecardeur [19] emphasized that online gaming addiction should be differentiated from IA because players do not report the experience of being on the internet when they are playing. Similarly, Spekman, Konijn, Roelfsma and Griffiths [28] pointed out that problematic gaming should be clearly distinguished from high game exposure, which may indicate enthusiasm in some players but psychopathology in others.

Cybersex was the second most researched cyberaddiction found in this analysis, together with social networking sites. Cybersex addiction has been associated mainly with excessive online pornographic consumption [18, 29-35], and risk factors have been associated mostly with the gratification hypothesis (i.e., reinforcement, learning mechanisms, and craving) 
[29]. Like online gaming addiction, cybersex addiction has been associated with personality and psychopathologic factors (e.g., neuroticism, agreeableness, conscientiousness, and obsessional checking) [30] and cognitive processes (i.e., decision-making, working process memory) [31, 32], although these are slightly different from those found in gaming addiction (in terms of the nature of this behavioral problem, such as cybersex and partners [33], or its relation to sexual abuse [34]). Furthermore, the cybersex treatments reported seem to be more varied $[35,36]$, using several classic modalities (e.g., psychodynamics, CBT, 12-step programs, group work, couples therapy) as well as contemporary ones (e.g., group analytic therapy for compulsive pornography users, behavioral strategies for cyberactivity).

On the other hand, excessive use of social networking sites represents quite a different cyberaddiction, with research into this practice focusing mainly on its negative impact on social well-being (e.g., social isolation, loneliness, interpersonal distress) [37]. The term cyber-relationship addiction has been proposed to place the social networking cyberaddiction within the range of cyber-relationship addictions, to emphasize the relevance of its psychosocial component $[16,38]$. The most researched social network is Facebook, especially with regard to motivational and psychological factors $[39,40]$, with studies showing negative consequences (e.g., addictive symptomatology such as salience, loss of control, and withdrawal) [39]. Studies also have investigated risk factors of excessive social networking sites use (e.g., low Internet self-efficacy, outcome expectancies, high impulsivity trait) [41]. This cyberaddiction has been studied the most in adolescents in Europe [42, 43].

Online (or Internet) gambling has increased rapidly and has been studied most often in adolescents. Those who gambled online represented a minor proportion of all gamblers, although they had a higher probability of developing an addiction [44, 45]. With regard to online gambling predictors, adolescents who gambled online increased their risk of generalized IA almost daily [43, 45]; most were males who gambled a wagered amount and grew up in a gambling family environment [44] with emotional and behavioral maladjustments [45]. Among the adults studied, most were men who gambled alone for more than $4 \mathrm{~h}$ per session in two or more online gambling activities, presenting comorbidity with other drug addictions (e.g., tobacco and alcohol) and commonalities with offline gamblers [46]. Finally, Internet gambling disorder (similar to IGD, as proposed by the APA) has been considered a behavioral addiction similar to compulsive buying on the Internet [47].

Online shopping addiction, also known as Internet shopaholism, represents the newest cyberaddiction and is similar to online gambling because a significant amount of money and time are spent online [48]. The scarce research conducted demonstrated specific psychological factors of motivation (e.g., exhaustive offers, immediate feelings) and a loss of control (e.g., low self-regulation), as well as addiction predictors differing only slightly from those of other specific cyberaddictions (i.e., female gender, negative emotional state, social anonymity, and cognitive overload) $[48,49]$.

\section{Main Findings of Clinical Studies on Internet Addiction}

Clinical research on IA follows three main themes. The most prevalent is the intervention used, followed by the associated symptomatology and the related diagnosis.

The treatment used most commonly in IA has been a specialized variant of CBT, namely CBT-IA [50], a 12-week three-phase approach including behavior modification to control compulsive internet use, cognitive restructuring related to excessive internet use, and harm reduction techniques for patients with comorbidities. However, alternative interventions have flourished recently. The psychodynamic approach appears useful for processes of attachment and individuation [51], balancing real relationships and virtual identities in adolescents and children. Similarly, developmental therapy improves IA in adolescents through an integrated approach involving their growth ambience (family) and technologies (commonly used). It focuses on investigating the affective and symbolic meanings behind the patient's internet behavior, which has been serving as a defense mechanism against his or her anxiety [52]. Group counseling also has proven effective in improving IA symptomatology and reducing the time spent online by adults [53]. Furthermore, therapeutic management combines CBT with the motivational interview [54], which also is used commonly in drug addiction interventions with adults.

Currently, IA is associated with a diverse group of psychosocial and psychopathologic symptoms related to a negative lifestyle, such as depression, anxiety, withdrawal, sleep disturbances, low self-esteem, poor body image and self-perception, narcissism, shyness, loneliness, aggression, negative affect, impulsivity, obsession and compulsion, interpersonal sensitivity, anxious attachment styles, disruptions in family functioning, distress, and poor coping skills.

Recent clinical research with IA patients found that the most frequently used test to diagnose IA (i.e., the Young IA test (IAT)) does not appear to classify them accurately (e.g., in Korea, the mean IAT score was 62.8 [55] and in Switzerland 52.9 [56], not achieving 70 of 100). A need exists for official diagnostic criteria to determine clinical severity; better screening instruments to avoid false diagnoses, including comorbidities (Axis I and II disorders) [57]; involvement of family members in the treatment; and follow-up studies to investigate the duration of this illness.

\section{Main Findings of Studies on the Prevalence of Internet Addiction}

Research on IA prevalence consists mainly of cross-sectional surveys completed by adolescents or young adults from 
specific countries in Europe or Asia; little cross-cultural research exists [43]. These studies used the IAT (or its adaptations) to estimate the prevalence of potential IA, and despite the use of the same instrument, the ranges vary widely (from $0.9 \%$ [58] to $11.8 \%$ [59]). However, other tests are starting to be used, including the Revised Generalized and Problematic Internet Use Scale (GPIUS2) [60] and the Compulsive Internet Use Scale (CIUS) [61]. Recently, new techniques have been applied to estimate prevalence, such as latent class analysis [62]. Lastly, with regard to risk factors, common characteristics such as time spent on the Internet, online gaming, social applications, mood regulation, and desire thinking appear to increase the probability of IA, whereas other sociodemographic factors, such as gender, age, immigration, marriage, and employment status, do not.

\section{Main Findings of Studies on the Contextual Factors of Internet Addiction}

Studies on the contextual factors of IA aimed to identify external factors that moderate or increase the risk of IA, especially in adolescents. The main contextual factors are kinship (e.g., parental behaviors such as control [63], style, support, and bonding, as well as emotional involvement and functioning in the family), school (e.g., cyberbullying [64]), work (stress as demand-control support and effort-reward imbalance [65]), social support (e.g., social interaction and ties [66]), and religion (e.g., god attachment [67], spiritual struggles).

\section{Main Findings on Internet Addiction Conceptualization}

The scientific conceptualization of the IA problem is progressing [68] with respect to whether to identify it as a mental disorder. Theoretical literature has been published examining the phenomenon from broader perspectives $[69,70]$, as well as empiric testing models such as the addiction component model by Griffiths [71], and demonstrating distinct nosologic entities between general IA and a specific IA, such as online gaming addiction [12, 72]. Other works have noted the lack of a conceptual framework, proposing their own rationale [73] or aggregating the psychosocial perspective to provide a multifactorial approach to IA [16].

\section{Main Findings of Psychometric Studies on Internet Addiction}

The psychometric studies centered on measuring IA through three approaches. First, the best items for an IA scale were selected by using psychometric techniques such as item response analysis [74] and careless and random responding [75]. Second, the psychometric properties (dimensionality, reliability, and validity) were tested for several IA scales, including the IAT [76-78], which showed an inconsistent factor structure, and the Revised Chen Internet Addiction Scale (CIAS-R) [79], the only one clinically validated. Third, many studies with cultural adaptations were published, such as the Problematic Internet Use Questionnaire (PIUQ) in French [80] and the IAT in Portuguese [81] and Greek [82]. Finally, the variety of options for psychometric analysis from specific adaptations constitutes another step toward validating IA measurement tools (e.g., the Italian GPIUS2 [83], the German CIUS [84]).

\section{Main Findings of Neuropsychological Studies on Internet Addiction}

The neuropsychological studies of IA are quite diverse and usually based on reviews $[85,86]$. These reports summarize cue reactivity, craving, and decision-making as key concepts in IA, and neuroimaging - such as magnetic resonance imaging (MRI), especially functional MRI and proton magnetic resonance - provides evidence revealing functional and structural abnormalities in the brain. Furthermore, the same brain reactions in IA are found in substance addiction and pathologic gambling $[86,87]$.

\section{Discussion}

The aim of this review is to observe the evolution of IA from the advent of IGD, covering its general and specific types [88 ${ }^{\circ}$. Based on PsycINFO data, in the 20-month period analyzed here, there was a steady increase in the number of scientific papers on IA published in peer-reviewed journals. According to bibliometric characteristics, several journals focusing on behavioral addictions were launched quite recently (e.g., Journal of Behavioral Addictions in 2012 and Cyberpsychology, Behavior, and Social Networking in 1998), and more-established journals related to the use of different technologies from a psychological or addictive perspective began focusing more on IA (e.g., Computers in Human Behavior from 1985 and Addictive Behaviors from 1975). Almost all these publications have a high-impact factor $(\sim 2.4)$ for the field of psychology. Moreover, the studies tend to be empiric and quantitative, with adult or adolescent samples, and mainly use the survey method classified in diverse themes (e.g., behavior and psychological disorders, addiction, testing).

With regard to the topics that emerged from analyzing the content of the IA literature, except for IGD, most focused on the generalized form and its different facets, although $28.5 \%$ concentrated on the spectrum of specific cyberaddictions. Recently, researchers noted that each type of IA is unique $[17,72]$. Although both types share similarities (e.g., addictive symptomatology and the medium through which the addiction 
occurs $[14 \bullet, 89])$, there clearly are differences (e.g., patterns of cognition and usage, personality traits, comorbidities, maladjusted behaviors) that clearly distinguish them from each other (e.g., online gaming addiction vs. online shopping addiction) from a psychosocial perspective. The other topics emerging in generalized IA are diverse because the research focused on its measurement and clinical and etiologic implications (considering internal and external factors), as well as on its nature, with the conceptualization issue still under debate in the behavioral addiction field $[12,69,70,72]$ and extended to IGD $[9-13,90]$. The field of IA continues to evolve [68], but not without controversy and a variety of perspectives $[12,16,90]$. However, the latest studies in this field have begun to embrace new and original approaches in clinical [91] and epidemiologic studies (e.g., cross-cultural studies [92], meta-analyses across nations [93]), among other scientific investigations.

\section{Limitations}

This review has several limitations. First, it focused on only one scientific database, albeit the most important one in the domain of psychology, and the period studied - one and a half years since the advent of IGD - may have been too short to observe the impact of IGD based on the IA articles analyzed, only about one fifth of which mention IGD. Second, the terms and Boolean operators selected for the search equation as well as the strategy of selecting specific criteria from the PsycINFO categories of refined search (i.e., "Narrow results by" section) resulted in these concrete results and the surfacing of this IA evolution from IGD advent. Third, the classification system used in the content analysis helped in identifying specific cyberaddictions at the cost of diminishing other categories (i.e., psychometric studies). Therefore, other potential specific cyberaddictions, such as online workaholism, were excluded. Finally, because of the large number of papers automatically and manually retained, only a superficial exploration was performed, without the opportunity to enter the subject more deeply because of the risk of unbalanced findings.

\section{Future Studies}

With regard to the bibliometric analysis, a new systematic review should be performed. It is suggested including other appropriate scientific databases (e.g., MEDLINE, Web of Science) and lengthening the study period to trace the parallel evolution of the cyberaddictive spectrum, including general and specific subtypes, and the body of IGD literature. Moreover, other search strategies might be followed using key words related to this field of behavioral technologic addiction. If the IA literature continues to grow, research on specific cyberaddictions will increase and new instruments and technologies, as well as clinical applications, will be tested. This may be an opportunity for a future review that delves deeper into the IA topics presented here, such as a specific cyberaddiction, like online gambling, observing psychological facets based on taxonomy (e.g., online poker gambling, online sports betting [94]). Other parameters related to bibliometric productivity (e.g., journals, authors, institutions, countries), as well as IA types, might be used.

\section{Conclusions}

Although IA still is the term applied in the study of technologic addiction problems, it remains unclear, and like its predecessor, the IGD construct currently is being debated. The lessons learned from this analysis are as follows: (i) as research lines have diversified and specialized, the focus of IA research has begun to shift from a more generalized construct in several domains (i.e., epidemiologic, clinical, and neuropsychological) to specific online addictive behaviors. (ii) From a psychological perspective, there seems to be a clear heterogeneity within the specific cyberaddiction spectrum, with an emphasis on online gaming, cybersex, and social networking. (iii) IGD had a slight impact on the IA scientific literature published by experts in psychology during the period under research, and most of the papers found were comments related to the APA proposal in DSM-5; however, this likely will change drastically in the rest of 2015. Research in IA seems to be in its adolescence after two decades of research, with controversies, new approaches, and several advancements, making this a rich field from a scientific perspective and a necessary field from a clinical and quality-of-life standpoint.

Acknowledgments This work was supported by a grant from the European Commission ("Tech Use Disorders"; Grant ID: FP7-PEOPLE2013-IEF-627999) awarded to Olatz Lopez-Fernandez.

\section{Compliance with Ethics Guidelines}

Conflict of Interest Olatz Lopez-Fernandez declares no conflict of interest.

Human and Animal Rights and Informed Consent This article does not contain any studies with human or animal subjects performed by any of the authors.

\section{References}

Papers of particular interest, published recently, have been highlighted as:

- Of importance

•. Of major importance

1. Young K, de Nabuco Abreu C. Internet addiction: a handbook and guide to evaluation and treatment. Hoboken, New Jersey: John Wiley \& Sons; 2011. This handbook, the first to address the conceptual and psychological factors associated with IA, was written by leading IA researchers. 
2. Griffiths MD. Technological addictions. Clin Psychol Forum. 1995;76:14-9. This study, the first to address technologic behavioral addiction, has inspired many studies on cyberaddictions.

3. Carbonell X, Guardiola E, Beranuy M, Bellés A. A bibliometric analysis of the scientific literature on Internet, video games, and cell phone addiction. J Med Libr Assoc. 2009;97:102-7.

4. American Psychiatric Association (APA). Diagnostic and statistical manual of mental disorders (DSM-5). Arlington, VA: Author; 2013. The latest DSM from the APA includes IGD in section III of its appendix.

5.• Petry NM, O'Brien CP. Internet gaming disorder and the DSM-5. Addiction. 2013;108(7):1186-7. This brief editorial, the first article to address the inclusion of IGD in the DSM-5, clarifies its role in facilitating research.

6.•• Petry NM, Rehbein F, Gentile DA, Lemmens JS, Rumpf H, Mößle $\mathrm{T}$, et al. An international consensus for assessing Internet gaming disorder using the new DSM-5 approach. Addiction. 2014;109(9): 1399-406. This international paper discusses the consensus on IGD research (conceptualizing and proposing a method to evaluate IGD with items associated with the nine criteria, translated in different languages to facilitate cross-cultural research).

7. Subramaniam M. Re-thinking Internet gaming: from recreation to addiction. Addiction. 2014;109(9):1407-8.

8. Goudriaan AE. Stepping up the game. Addiction. 2014;109(9): 1409-11.

9. Ko C, Yen J. The criteria to diagnose Internet gaming disorder from causal online gamer. Addiction. 2014;109(9):1411-2.

10. Kardefelt-Winther D. Meeting the unique challenges of assessing Internet gaming disorder. Addiction. 2014;109(9):1568-70.

11. Griffiths MD, King DL, Demetrovics Z. DSM-5 Internet gaming disorder needs a unified approach to assessment. Neuropsychiatry. 2014;4(1):1-4.

12. Griffiths MD, Pontes HM. Internet addiction disorder and Internet gaming disorder are not the same. J Addict Res Ther. 2014;5:e124.

13. Pontes HM, Griffiths MD. Assessment of Internet gaming disorder in clinical research: past and present perspectives. Clin Res Regul Aff. 2014;31(2-4):35-48.

14. Griffiths MD, Szabo A. Is excessive online usage a function of medium or activity? An empirical pilot study. J Behav Addict. 2014;3(1):74-7. This empiric study investigated whether Internet excessive use is associated with a medium or an activity, a current debate in the technologic behavioral addictions literature.

15. Petry NM, Rehbein F, Gentile DA, Lemmens JS, Rumpf H, Mößle $\mathrm{T}$, et al. Moving Internet gaming disorder forward: a reply. Addiction. 2014;109(9):1412-3. This reply to comments made by several IA researchers regarding the consensual proposal of IGD conceptualization and measurement offers some clarification of IGD.

16. Suissa AJ. Cyberaddictions: toward a psychosocial perspective. Addict Behav. 2014;39(12):1914-8.

17. Laconi S, Tricard N, Chabrol H. Differences between specific and generalized problematic Internet uses according to gender, age, time spent online and psychopathological symptoms. Comput Hum Behav. 2015;48:236-44.

18. Kor A, Zilcha-Mano S, Fogel YA, Mikulincer M, Reid RC, Potenza MN. Psychometric development of the Problematic Pornography Use Scale. Addict Behav. 2014;39(5):861-8.

19. Lecardeur L. Psychopathologie du jeu multi-joueurs en ligne. Ann Med Psychol. 2013;171(8):579-86.

20. Cole SH, Hooley JM. Clinical and personality correlates of MMO gaming: anxiety and absorption in problematic Internet use. Soc Sci Comput Rev. 2013;31(4):424-36.

21. Li H, Wang S. The role of cognitive distortion in online game addiction among Chinese adolescents. Child Youth Serv Rev. 2013;35(9):1468-75.
22. Kuss DJ. Internet gaming addiction: current perspectives. Psychol Res Behav Manag. 2013;6:125-37.

23. Hilgard J, Engelhardt CR, Bartholow BD. Individual differences in motives, preferences, and pathology in video games: the gaming attitudes, motives, and experiences scales (GAMES). Front Psychol. 2013;4:1-13.

24. Bailey K, West R, Kuffel J. What would my avatar do? Gaming, pathology, and risky decision making. Front Psychol. 2013;4:1-10.

25. Metcalf O, Pammer K. Impulsivity and related neuropsychological features in regular and addictive first person shooter gaming. Cyberpsychol Behav Soc Netw. 2014;17(3):147-52.

26. Kneer J, Rieger D, Ivory JD, Ferguson C. Awareness of risk factors for digital game addiction: interviewing players and counselors. Int J Ment Heal Addict. 2014;12(5):585-99.

27. Marco C, Chóliz M. Tratamiento cognitivo-conductual de la adicción a videojuegos de rol online: Fundamentos de propuesta de tratamiento y estudio de caso. Anales de Psicología. 2014;30(1): $46-55$.

28. Spekman MLC, Konijn EA, Roelofsma PHMP, Griffiths MD. Gaming addiction, definition and measurement: a large-scale empirical study. Comput Hum Behav. 2013;29(6):2150-5.

29. Laier C, Pawlikowski M, Pekal J, Schulte FP, Brand M. Cybersex addiction: experienced sexual arousal when watching pornography and not real-life sexual contacts makes the difference. J Behav Addict. 2013;2(2):100-7.

30. Egan V, Parmar R. Dirty habits? Online pornography use, personality, obsessionality, and compulsivity. J Sex Marital Ther. 2013;39(5):394-409.

31. Laier C, Schulte FP, Brand M. Pornographic picture processing interferes with working memory performance. J Sex Res. 2013;50(7):642-52.

32. Laier C, Pekal J, Brand M. Cybersex addiction in heterosexual female users of internet pornography can be explained by gratification hypothesis. Cyberpsychol Behav Soc Netw. 2014;17(8):505-11.

33. Ballester-Arnal R, Castro-Calvo J, Gil-Llario M, GiménezGarcía C. Relationship status as an influence on cybersex activity: cybersex, youth, and steady partner. J Sex Marital Ther. 2014;40(5):444-56.

34. Chaney MP, Burns-Wortham C. The relationship between online sexual compulsivity, dissociation, and past child abuse among men who have sex with men. J LGBT Issues Couns. 2014;8(2):146-63.

35. Woods J. Group analytic therapy for compulsive users of internet pornography. Psychoanal Psychother. 2013;27(4):306-18.

36. Riemersma J, Sytsma M. A new generation of sexual addiction. Sex Addiction Compulsivity. 2013;20(4):306-22.

37. Baek YM, Bae Y, Jang H. Social and parasocial relationships on social network sites and their differential relationships with users' psychological well-being. Cyberpsychol Behav and Soc Netw. 2013;16(7):512-17.

38. Tzavela EC, Mavromati FM. Online social networking in adolescence: associations with development, well-being and internet addictive behaviors. Int $\mathrm{J}$ Child Adolesc Health. 2013;6(4):411-20.

39. Balakrishnan V, Shamim A. Malaysian Facebookers: motives and addictive behaviors unraveled. Comput Hum Behav. 2013;29(4): 1342-9.

40. Uysal R, Satici SA, Akin A. Mediating effect of Facebook ${ }^{\circledR}$ addiction on the relationship between subjective vitality and subjective happiness. Psychol Rep. 2013;113(3):948-53.

41. Wu AMS, Cheung VI, Ku L, Hung EPW. Psychological risk factors of addiction to social networking sites among Chinese smartphone users. J Behav Addict. 2013;2(3):160-6. 
42. De Cock R, Vangeel J, Klein A, Minotte P, Rosas O, Meerkerk G. Compulsive use of social networking sites in Belgium: prevalence, profile, and the role of attitude toward work and school. Cyberpsychol Behav Soc Netw. 2014;17(3):166-71.

43. Tsitsika AK, Tzavela EC, Janikian M, Ólafsson K, Iordache A, Schoenmakers TM, et al. Online social networking in adolescence: patterns of use in six European countries and links with psychosocial functioning. J Adolesc Health. 2014;55(1):141-7.

44. Wong ILK, So EMT. Internet gambling among high school students in Hong Kong. J Gambl Stud. 2014;30(3):565-76.

45. Critselis E, Janikian M, Paleomilitou N, Oikonomou D, Kassinopoulos M, Kormas G, et al. Internet gambling is a predictive factor of Internet addictive behavior among Cypriot adolescents. J Behav Addict. 2013;2(4):224-30.

46. McCormack A, Shorter GW, Griffiths MD. Characteristics and predictors of problem gambling on the Internet. Int J Ment Heal Addict. 2013;11(6):634-57.

47. Fawcett J. The human condition and behavioral addictions. Psychiatr Ann. 2014;44(8):362.

48. Duroy D, Gorse P, Lejoyeux M. Characteristics of online compulsive buying in Parisian students. Addict Behav. 2014;39(12):182730.

49. Rose S, Dhandayudham A. Towards an understanding of Internetbased problem shopping behavior: the concept of online shopping addiction and its proposed predictors. J Behav Addict. 2014;3(2): 83-9.

50. Young KS. Treatment outcomes using CBT-IA with Internetaddicted patients. J Behav Addict. 2013;2(4):209-15.

51. Bilke-Hentsch O, Seiffge-Krenke I, Stoll M, te Wildt B. Pathologischer Internet- und Medienkonsum bei Jugendlichen und Heranwachsenden: Psychodynamische Aspekte in der klinischen Konzeptbildung. PDP Psychodynamische Psychotherapie: Forum der tiefenpsychologisch fundierten Psychotherapie. 2013;12(2):81-90.

52. Lancini M, Cirillo L. Il trattamento delle dipendenze da Internet in adolescenza. Psichiatria e Psicoterapia. 2013;32(2):89-100.

53. Liu D, Lu N, He J, Tang H, Zhou L. Effects of internet addiction group counseling on Internet using and study management in college students. Chin Ment Health J. 2013;27(7):496-501.

54. Chele GE, Macarie G, Stefanescu C. Management of Internet addictive behaviors in adolescents. Int $\mathrm{J}$ Child Adolesc Health. 2013;6(4):443-53

55. Kim SJ, Park D, Ryu S, Yu J, Ha JH. Usefulness of Young's Internet addiction test for clinical populations. NordJournal Psychiatry. 2013;67(6):393-9.

56. Thorens G, Achab S, Billieux J, Khazaal Y, Khan R, Pivin E, et al. Characteristics and treatment response of self-identified problematic Internet users in a behavioral addiction outpatient clinic. J Behav Addict. 2014;3(1):78-81.

57. Floros G, Siomos K, Stogiannidou A, Giouzepas I, Garyfallos G. Comorbidity of psychiatric disorders with Internet addiction in a clinical sample: the effect of personality, defense style and psychopathology. Addict Behav. 2014;39(12):1839-45.

58. Naffise M, Mohammad A, Ahmad PB, Omid R, Ayatollahi A, Reza $\mathrm{B}$, et al. The prevalence of Internet addiction among the students of Rafsanjan University of Medical Sciences. ASEAN J Psychiatry. 2013;14(2):109-16.

59. Yadav P, Banwari G, Parmar C, Maniar R. Internet addiction and its correlates among high school students: a preliminary study from Ahmedabad. India Asian J Psychiatry. 2013;6(6):500-05.

60. Gámez-Guadix M, Orue I, Calvete E. Evaluation of the cognitivebehavioral model of generalized and problematic Internet use in Spanish adolescents. Psicothema. 2013;25(3):299-306.

61. Kuss DJ, van Rooij AJ, Shorter GW, Griffiths MD, van de Mheen D. Internet addiction in adolescents: prevalence and risk factors. Comput Hum Behav. 2013;29(5):1987-96.
62. Rumpf H, Vermulst AA, Bischof A, Kastirke N, Gürtler D, Bischof $\mathrm{G}$, et al. Occurrence of Internet addiction in a general population sample: a latent class analysis. Eur Addict Res. 2014;20(4):8.

63. Li X, Li D, Newman J. Parental behavioral and psychological control and problematic Internet use among Chinese adolescents: the mediating role of self-control. Cyberpsychol Behav Soc Netw. 2013;16(6):442-7.

64. Burnett AL, Yozwiak JA, Omar HA. Cyberbullying: a brief review. Int J Child Adolesc Health. 2013;6(4):465-8.

65. Chen S, Gau SS, Pikhart H, Peasey A, Chen S, Tsai M. Work stress and subsequent risk of Internet addiction among information technology engineers in Taiwan. Cyberpsychol Behav Soc Netw. 2014;17(8):542-50.

66. Wang ES, Wang MC. Social support and social interaction ties on Internet addiction: integrating online and offline contexts. Cyberpsychol Behav Soc Netw. 2013;16(11):843-9.

67. Knabb JJ, Pelletier J. The relationship between problematic Internet use, God attachment, and psychological functioning among adults at a Christian university. Ment Health Relig Cult. 2014;17(3):239 51.

68. Tsitsika A, Janikian M, Greydanus DE, Omar HA, Merrick J. A new millennium: a new age of behavioral disorders? Int J Child Adolesc Health. 2013;6(4):363-6.

69. Hsu S, Shih H. Is Internet addiction a mental disorder? A review of empirical and conceptual studies. Bull Educ Psychol. 2013;44(4): 773-92.

70. Spada MM. An overview of problematic internet use. Addict Behav. 2014;39(1):3-6.

71. Kuss DJ, Shorter GW, van Rooij AJ, Griffiths MD, Schoenmakers TM. Assessing Internet addiction using the parsimonious Internet addiction components model — a preliminary study. Int J Ment Heal Addict. 2014;12(3):351-66.

72. Rehbein F, Mößle T. Video game and Internet addiction: is there a need for differentiation? Sucht: Zeitschrift für Wissenschaft und Praxis. 2013;59(3):129-42.

73. Moreno MA, Jelenchick LA, Christakis DA. Problematic internet use among older adolescents: a conceptual framework. Comput Hum Behav. 2013;29(4):1879-87.

74. Zhang J, Xin T. Measurement of Internet addiction: an item response analysis approach. Cyberpsychol Behav Soc Netw. 2013;16(6):464-8.

75. Meyer JF, Faust KA, Faust D, Baker AM, Cook NE. Careless and random responding on clinical and research measures in the addictions: a concerning problem and investigation of their detection. Int J Ment Heal Addict. 2013;11(3):292-306.

76. Lai C, Mak K, Watanabe H, Ang RP, Pang JS, Ho RCM. Psychometric properties of the Internet addiction test in Chinese adolescents. J Pediatr Psychol. 2013;38(7):794-807.

77. Watters CA, Keefer KV, Kloosterman PH, Summerfeldt LJ, Parker JDA. Examining the structure of the Internet Addiction Test in adolescents: a bifactor approach. Comput Hum Behav. 2013;29(6):2294-302.

78. Kern L, Acier D. Adaptation française de l'échelle Problematic Internet Use Questionnaire. L'Évolution Psychiatrique. 2013;78(3):357-71.

79. Mak K, Lai C, Ko C, Chou C, Kim D, Watanabe H, et al. Psychometric properties of the Revised Chen Internet Addiction Scale (CIAS-R) in Chinese adolescents. J Abnorm Child Psychol. 2014;42(7):1237-45.

80. Sung M, Shin Y, Cho S. Factor structure of the Internet Addiction Scale and its associations with psychiatric symptoms for Korean adolescents. Community Ment Health J. 2014;50(5):612-8.

81. Pontes HM, Patrão IM, Griffiths MD. Portuguese validation of the Internet Addiction Test: an empirical study. J Behav Addict. 2014;3(2):107-14. 
82. Tsimtsiou Z, Haidich A, Kokkali S, Dardavesis T, Young KS, Arvanitidou M. Greek version of the Internet Addiction Test: a validation study. Psychiatry Q. 2014;85(2):187-95.

83. Fioravanti G, Primi C, Casale S. Psychometric evaluation of the Generalized Problematic Internet Use Scale 2 in an Italian sample. Cyberpsychol Behav Soc Netw. 2013;16(10):761-6.

84. Guertler D, Broda A, Bischof A, Kastirke N, Meerkerk G, John U, et al. Factor structure of the Compulsive Internet Use Scale. Cyberpsychol Behav Soc Netw. 2014;17(1):46-51.

85. Qin Y, Liu J, Zhou S. Advancement in the research on magnetic resonance imaging of Internet game addiction. Chinese J Clin Psychol. 2013;21(3):376-8.

86. Brand M, Laier C. Neuropsychologic der pathologischen Internetnutzung. Sucht: Zeitschrift für Wissenschaft und Praxis. 2013;59(3):143-52.

87. Feng Q, Chen X, Sun J, Zhou Y, Sun Y, Ding W, et al. Voxel-level comparison of arterial spin-labeled perfusion magnetic resonance imaging in adolescents with Internet gaming addiction. Behav Brain Funct. 2013;9:1-11.

88. Davis RA. A cognitive behavioral model of pathological Internet use. Comput Hum Behav. 2001;17:187-95. The authors present the first theoretic model to study IA, proposing the classification of generalized and specialized subtypes. Their work has inspired many studies on problematic internet use, as well as scales to measure it, and, as noted in this review, might support the advancement of cyberaddiction in general (and IGD specifically) within the specified subtype.

89. Adiele I, Olatokun W. Prevalence and determinants of Internet addiction among adolescents. Comput Hum Behav. 2014;31:100-10.

90. Van Rooij AJ, Prause N. A critical review of "Internet addiction" criteria with suggestions for the future. J Behav Addict. 2014;3(4): 203-13.

91. Graham Jr J. Narrative therapy for treating video game addiction. Int J Ment Heal Addict. 2014;12(6):701-7.

92. Blinka L, Škařupová K, Ševčíková A, Wölfling K, Müller KW, Dreier M. Excessive internet use in European adolescents: what determines differences in severity? International Journal of Public Health 2014

93. Cheng C, Yee-lam LA. Internet addiction prevalence and quality of (real) life: a meta-analysis of 31 nations across seven world regions. Cyberpsychol Behav Soc Netw. 2014;17(12):755-60.

94. Matthews N, Farnsworth B, Griffiths MD. A pilot study of problem gambling among student online gamblers: mood states as predictors of problematic behavior. CyberPsychol Behav. 2009;12(6):741-5. 